\title{
Red de alumnos normalistas trabajando: pensar globalmente, actuar virtualmente
}

\author{
Baltazar Contreras Durán \\ Guadalupe Melina Núñez Valladárez \\ Jorge Antonio Alfaro Rivera
}

Estudiante del $8^{\circ}$ Semestre de la Licenciatura en Educación Secundaria Estudiante del $8^{\circ}$ Semestre de la Licenciatura en Educación Primaria Profesor investigador, Escuela Normal Superior Oficial de Guanajuato

\section{Resumen}

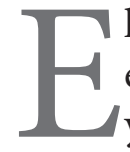

1 artículo expone una reflexión de dos estudiantes normalistas de Guanajuato y del Estado de México, pertenecientes a una red virtual de alumnos, como actividad que complementa su formación. Ésta es coordinada por un académico de la Escuela Normal Superior Oficial de Guanajuato con apoyo de cuatro docentes de Escuelas Normales del Estado de México y pretende favorecer el desarrollo de competencias digitales como futuros docentes. El grupo trabaja virtualmente en una plataforma ad hoc para ello, en uno de los mayores buscadores del orbe: Google. La red se denomina: "Red de Alumnos Normalistas Trabajando", título que tiene que ver con el propósito mencionado, basado en una perspectiva constructivista, donde profesores y estudiantes al interactuar entre sí, se convierten en monitores que favorecen una dinámica basada en el aprendizaje colaborativo. El uso de Google Groups y sus diversas herramientas posibilitan que aprendamos a usarlas de manera reflexiva y analítica, pues ahí se plasman diversos puntos de vista, propiciando el desarrollo de diversas competencias docentes en ambientes virtuales, proporcionando una alfabetización digital que permite utilizar de manera eficaz y eficiente estos nuevos instrumentos tecnológicos que constituyen las Tecnologías de la Información y la Comunicación (TIC), en actividades profesionales y personales.

Palabras clave: competencias digitales, Google Groups, análisis, reflexión 


\section{Introducción}

En el Informe Mundialdela UnESCO(2005) "Hacia las sociedades del conocimiento" se ha denominado la tercera revolución industrial a la llegada de las nuevas tecnologías de la información y la comunicación (NTIC) porque éstas han creado una nueva dinámica social, económica y cultural. Esto ha generado una constante evolución e interdependencia entre los diferentes elementos que conforman la sociedad, principalmente en el ámbito educativo.

Por ello, resulta conveniente lanzar una pregunta que constantemente se escucha entre los grupos de docentes ya formados y que se convierte en uno de las principales enigmas para todos aquéllos que tienen dificultades para avanzar a la par de los retos y cambios tecnológicos: ¿Cómo desarrollar competencias digitales en alumnos normalistas?

La respuesta desde luego es amplia y compleja, pero quizá aventurándonos un poco, a partir de las propuestas de nuestra red virtual, podríamos decir que la clave radica en el uso de herramientas tecnológicas y el desarrollo de las mismas, utilizándolas en grupos para realizar trabajos colaborativos en donde la diversidad de pensamientos contribuyan a enriquecer las temáticas, convirtiéndose en una necesidad para todos y cada uno de nosotros, pues en la medida en que comprendamos que los recursos digitales son una herramienta con el potencial de posibilitar el progreso del ser humano: desarrollando sus capacidades, habilidades, competencias, actitudes y valores (Schmelkes, 1994).

Consideramos de vital importancia el uso de estos medios, particularmente de un ins- trumento tan innovador como la Internet, pues nos está facilitando el acceso a recursos que hace pocos años no existían. Su desarrollo ha facilitado la creación de un espacio donde se puede participar en foros y conferencias, localizar información de lo más variado, expresar opiniones, comunicarse con otros usuarios, realizar compras desde casa, y un largo etcétera.

Ante ello, no podemos negar que los avances tecnológicos han aportado enormes beneficios a toda la humanidad, lo que aumenta las posibilidades para cambiar el mundo adecuándolo a nuestras necesidades. No obstante, en el informe publicado por la Organización para la Cooperación para el Desarrollo Económico (OCDE), en 1994 sobre calidad en la enseñanza, se confirman las limitaciones de las personas para acceder en el campo de las Tecnologías de la Información y las Comunicaciones (TIC), y recomienda a las autoridades educativas mexicanas generar estrategias para adaptarse a estas situaciones, estableciendo nuevos desafíos y demandas hacia las escuelas, docentes y alumnos, con ello surgen nuevas y ampliadas expectativas sobre las instituciones educativas (Fernández, 2003).

La vida de los seres humanos y la atención a sus necesidades van de la mano de la tecnología, y, ante esto, debemos saber hacer uso de ella y sacarle el beneficio necesario, como la participación dentro de espacios donde podamos comentar, confrontar y compartir información obtenida de diversos recursos contenidos en la Web acerca de la realidad y vida cotidiana; es decir, saber encontrar información para mejorar la calidad de la educación y no sólo tomarlo como un pasatiem- 
po. En este contexto, se creó la Red de Alumnos Normalistas Trabajando en un ambiente virtual (google groups).

El presente documento tiene el propósito de reflexionar acerca de las experiencias vividas, así como exponer las ventajas y desventajas en la formación de los futuros docentes miembros de este grupo. Las conclusiones aportan información que pudiera ser tomada en cuenta para el trabajo mismo de la red así como para investigaciones relacionadas con la temática.

\section{Nociones de competencia digital}

¿Qué son las competencias digitales? ¿Cuáles son? ¿Qué significa desarrollar competencias digitales? ¿Por qué es importante que un alumno normalista desarrolle estas competencias? ¿Cómo se adquieren? Consideramos necesario hacer una reflexión sobre lo que hoy en día significa una formación docente que ayude a desarrollar competencias digitales.

Partimos de la idea de que el término "competencia" es de carácter polisémico, su uso y aplicación dependen de la manera en que se conceptualiza en los distintos ámbitos. Con respecto a las competencias en el ámbito educativo, primeramente es importante tener presente que según el constructivismo, los contenidos son saberes que permiten afrontar diversas situaciones personales o sociales que se presenten. Como dice César Coll, son "el conjunto de saberes culturales cuya asimilación y apropiación por parte de los alumnos se considera esencial para su desarrollo y socialización” (1992: 155).

Con esto entendemos que una competencia no simplemente es desempeñarse mejor que todos en alguna situación, el término hace referencia a las habilidades intelectuales desarrolladas por un sujeto frente a un objetivo determinado, en el transcurso de su formación educativa y a lo largo de su vida.

Lo anterior exige de una nueva cultura que supone nuevas formas de ver y entender el mundo que nos rodea, el uso de nuevas herramientas o instrumentos y la implantación de nuevos valores y normas de comportamiento "Una competencia es el desarrollo de una capacidad para el logro de un objetivo o resultado en un contexto dado" (Bazdrech, 2005: 65). Esto refiere a la capacidad de la persona para dominar tareas específicas que le permitan solucionar las problemáticas que le plantea la vida cotidiana, como el que un estudiante aprenda a leer o que aprenda a contar el dinero para adquirir un producto en una tienda. Ahora bien, en la actualidad existen investigaciones (Chan, 2005; Hernández y González, 2005; López y Flores, 2006; Sobrado, 2006; Guitert, Romeau y Pérez-Mateo, 2007; UnEsCo, 2008), que relacionan a las competencias a desarrollar con el uso de las TIC.

Pero, qué competencias digitales debe desollar un docente en formación. Al respecto, López y Flores (2006), consideran importante que cualquier persona que viva en el siglo XXI desarrolle:

a) Competencias básicas en el uso de las TIC: es necesario principalmente aprender a trabajar utilizando las tecnologías. Saber acceder a cualquier sitio, ingresar a páginas web y resolver situaciones problemáticas usando las habilidades desarrolladas.

b) El uso de las Tic para la búsqueda y selección de la información: sin lugar a dudas, 
una de las grandes ventajas que las TIC nos aportan, es la cantidad de información que puede ser puesta de manera virtual a disposición de todos, pero nos encontramos en una situación en permanente revolución, ya que los sitios webs dedicados a la formación van creciendo y complejizándose por la diversidad de temáticas que van apareciendo. Situación que se presenta "desde una perspectiva institucional, como asociativa o personal" (Cabero y Llorente, 2008: 2), ofreciéndonos de esta forma, una amplitud de información con la que profesores y estudiantes podemos interaccionar tanto en el salón de clases, como en ambientes virtuales.

c) El uso de las Tic como medio de comunicación. Las TIC permiten que varias personas realicen actividades de interacción comunicativa independientemente del espacio y tiempo en el que cada uno se sitúe, pero en el ámbito educativo, permiten la colaboración e intercambio de información entre profesor y alumnos. El chat y el correo electrónico, son herramientas de comunicación que cada vez son más utilizadas, y tienen un potencial insospechado para los estudiantes.

d) El uso de las Tic como medio para el aprendizaje: con el enfoque adecuado, los estudiantes podemos acercarnos cada vez más al ideal de ser los propios diseñadores de nuestro proceso de aprendizaje, usando la tecnología como herramienta para analizar el mundo, acceder a la información, interpretar $y$ analizar el propio conocimiento y representar lo que sabemos. Si confrontamos esto con los tradicionales modelos de enseñanza centrados en el profesor, las TIC permiten pasar a modelos centrados en el estudiante, es decir, pasar de una cultura de la enseñanza, a una cultura del aprendizaje, ya que la mejor forma de aprender, no es reproduciendo conocimientos, sino construyéndolos (Cabero y Llorente, 2008).

Por tal motivo, consideramos que ser docentes en la sociedad actual, es algo más que ser un experto en las diversas asignaturas, pues se requiere ser un facilitador, un coordinador de experiencias de aprendizaje y promotor de estrategias que ayuden a los estudiantes a aprender cómo aprender de forma activa, participativa y con un cierto grado de autonomía que se fortalece cada vez más hasta llegar a que un estudiante sea responsable de sus propios procesos de aprendizaje (Andrade y Sánchez, 2010).

Creemos que los profesores siempre se han visto tentados a querer adaptar las tecnologías a sus prácticas tradicionales, pero esto es cada vez más difícil, sobre todo cuanto se trata de recursos como Internet, debido a su gran potencial interactivo y a la cantidad ilimitada de información a la que se tiene acceso. Los materiales didácticos y los demás recursos de apoyo a la educación a disposición de profesores y estudiantes se han multiplicado de manera exponencial.

De esta manera, a los tradicionales libros, juegos, vídeos y sus aportaciones, ahora se suman los materiales multimedia y las infinitas webs de Internet que facilitan al profesorado el acceso a la información sobre los temas de interés para su grupo. Por otra parte, un uso pedagógico adecuado de las TIC también propicia una mayor autonomía y calidad en los aprendizajes de los estudiantes, ya que además de facilitar información, canales de comunicación e instrumentos de productividad para un mejor proceso de la informa- 
ción, actúan como instrumentos cognitivos que pueden apoyar y expandir su capacidad de pensamiento, como es el caso de Google Groups.

\section{Google Groups: el elemento fundamental}

Tenemos la grata experiencia de pertenecer a un grupo en donde un docente de la Escuela Normal Superior Oficial de Guanajuato, nos ha dado la oportunidad de trabajar utilizando una plataforma en la que se hace uso de diversos recursos: el correo electrónico, el chat, los foros, debates, libros y archivos en la misma, el sitio: https:/groups. google.com/group/red-de-alumnos-normalistas-trabajando/about?hl=es (Figura 1) fue creado para intercambiar ideas, dar puntos de opinión, debatir sobre diversos temas de carácter educativo, elaborar mapas mentales a partir de lecturas, entre muchas otras cosas. Sin duda alguna, consideramos muy importante integrar la red en la plataforma, lo que demuestra nuestro interés por el uso de los recursos tecnológicos. Resulta interesante la manera en que se pueden integrar, logrando como producto la realización de competencias en el manejo de las TIC contenidas en la World Wide Web (www).

Figura 1. Página principal de Google Groups: "Red de alumnos normalistas trabajando"

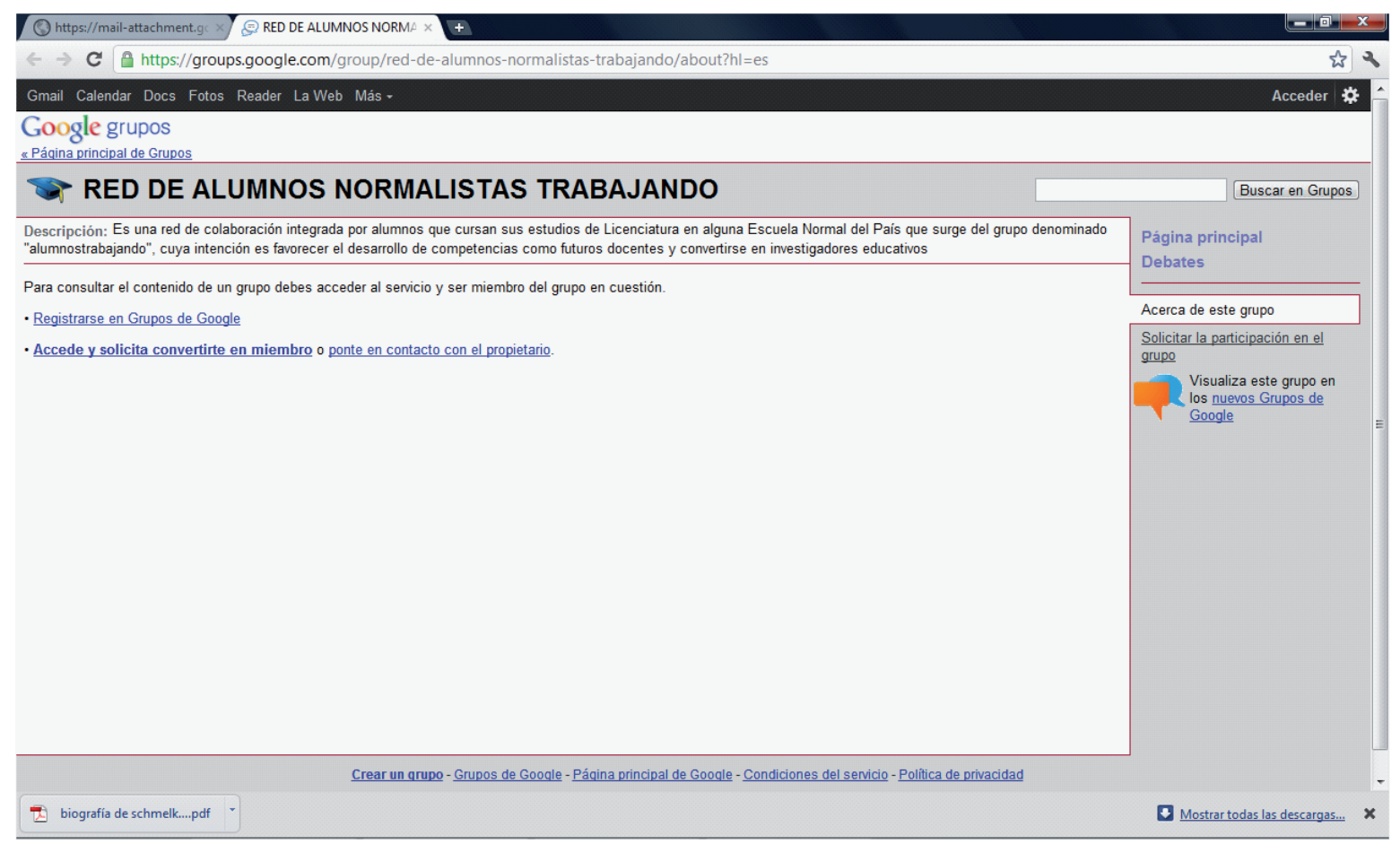


Una ventaja de singular importancia es que esta herramienta y plataforma es de servicio gratuito y de fácil acceso, requiere mínimas habilidades digitales, situación que favorece el desarrollo de actividades en este sitio tanto para el administrador y/o dueño como para los participantes. Los miembros pueden agregar la información que consideren importante y generar los debates, si el administrador del grupo se los permite. Además, la página cuenta con los siguientes apartados: debate en línea o por correo, estadísticas, notificaciones por correo, páginas para agregar información. Esto permite al usuario contar con diferentes elementos para realizar sus actividades.

La comunidad estaba conformada hasta el 28 de Julio de 2011, por siete docentes que coordinan el trabajo, dos alumnos administradores, 81 alumnos de dos escuelas Normales del Estado de México y cuatro alumnos de la Escuela Normal Superior Oficial de Guanajuato, con la posibilidad de incrementar la cantidad, ya que actualmente hay 43 alumnos pendientes con posibilidad de incorporarse a la red.

El trabajo en una plataforma virtual resultó de interés desde el principio para muchos de los involucrados, aunque con algo de temor por no estar familiarizados con la herramienta ya que en algunos casos, solamente se conocía Hi5, Facebook, Twitter, My Space y algunos blogs sociales donde se suele interactuar con otras personas, pero diseñados solo para platicar y comunicarse, conocer nuevos amigos; algo completamente diferente a lo que es la experiencia en una plataforma con fines educativos y de investigación.

El coordinador, con ayuda de dos admi- nistradores -de la Escuela Normal Superior Oficial de Guanajuato y de la Escuela Normal de Ecatepec-, ha guiado al grupo en el uso de herramientas tecnológicas con fines educativos (subir y descargar archivos para la lectura y para compartir opiniones e ideas). La red se formó a partir de una invitación que se envío a cuenta de correo para que participaran los alumnos normalistas. La "Red de alumnos normalistas trabajando", se define como: "una red de colaboración integrada por alumnos que cursan sus estudios de licenciatura en alguna Escuela Normal del país que surge del grupo denominado "alumnos trabajando", cuya intención es favorecer el desarrollo de competencias como futuros docentes y convertirse en investigadores educativos" (Figura 2).

Ya conformado el grupo, cada uno de los integrantes hizo una presentación personal en donde muestra su foto, lugar de nacimiento y algunos datos personales sobre lo que les gusta hacer, pasatiempos, ideales, metas, entre otros (Figura 3).

Desde que comenzó a operar la red se abrió un debate sobre diversos temas educativos, algunos propuestos por algún docente y otros por iniciativa de los administradores. La participación ha sido muy variada; algunos miembros constantemente participan, mientras que otros lo hacen con menos frecuencia. Se podría decir que hay actitudes de interacción, convivencia y aprendizaje en un medio virtual, pero con un enfoque colaborativo encaminado a la investigación educativa.

En esta red se sube y comparte información referente a investigación o educación (Figura 4); el coordinador y los docentes administradores están constantemente aten- 
Figura 2. Página de presentación del grupo

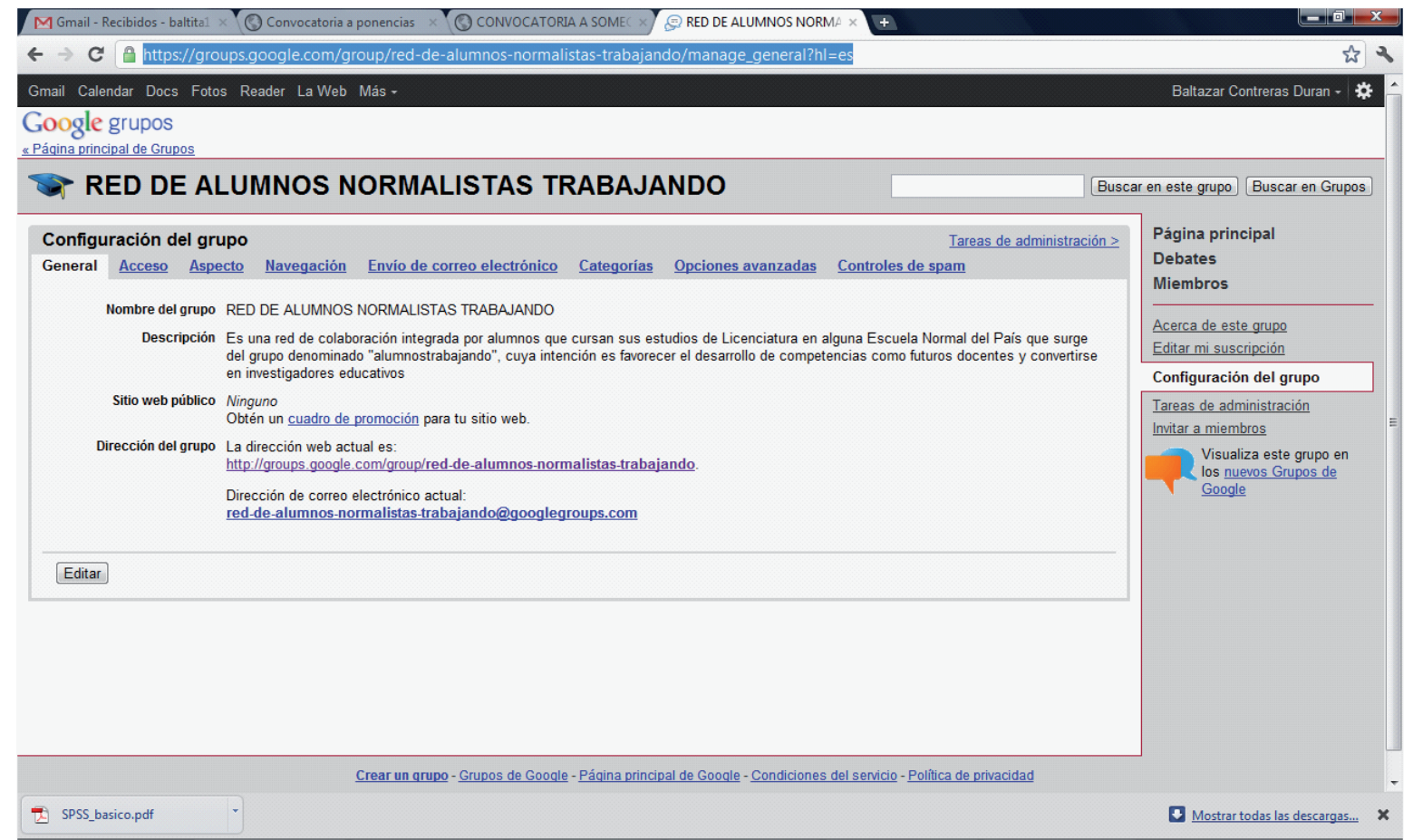

Figura 3. Página del grupo donde se muestran presentaciones de algunos miembros que conforman la"Red de alumnos normalistas trabajando"

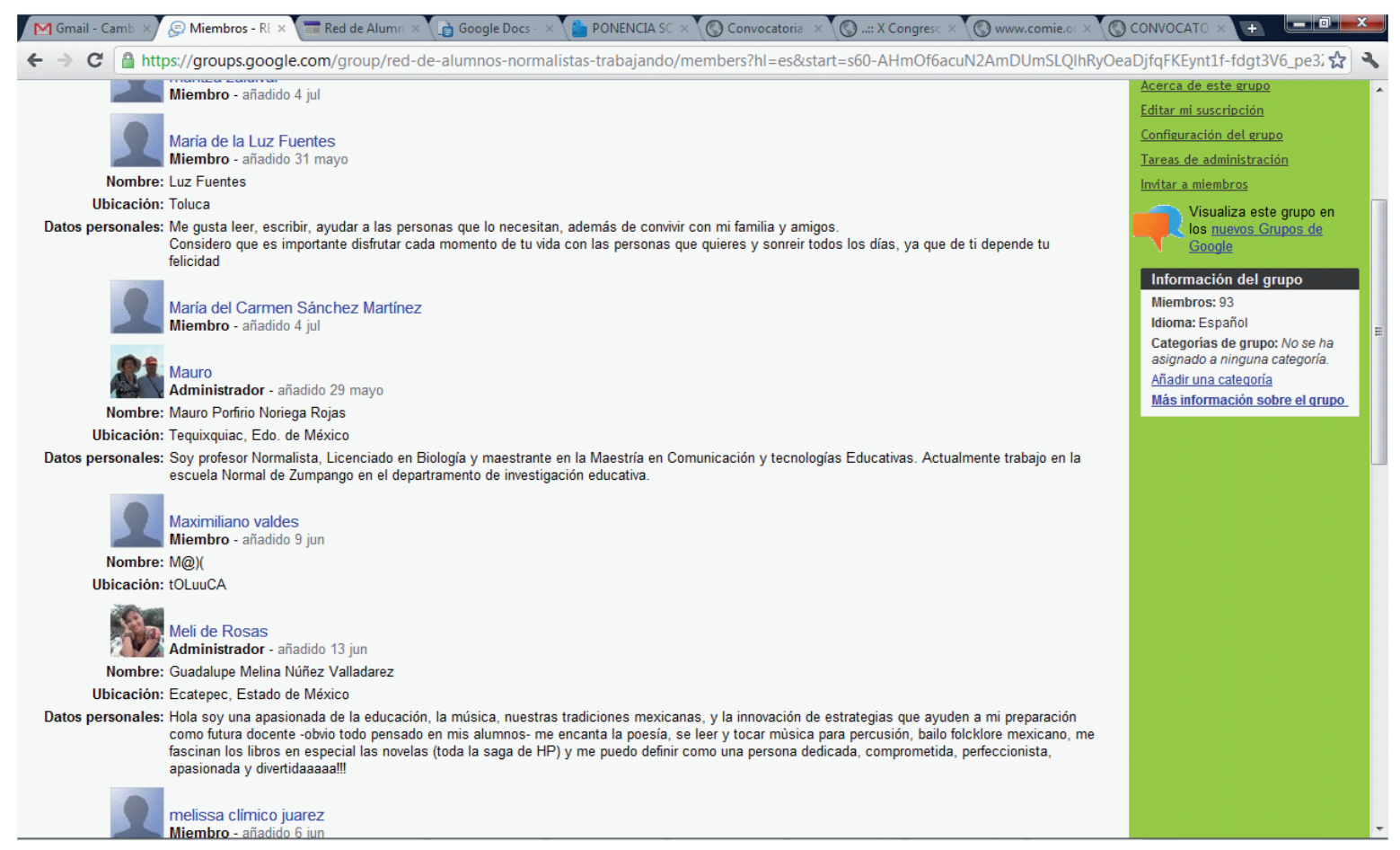


tos para aclarar dudas, hacer comentarios y sugerencias valiosas, que aportan elementos para desarrollar y mejorar el trabajo.

Esto ayuda para ir creciendo humana y académicamente, pues se observan y evalúan los trabajos de los demás compañeros y sirve para comparar lo realizado por cada uno de los miembros en cuanto a valorar el trabajo personal con un sentido profesional: aprender de y con los demás en un ambiente virtual; es decir, lo que realmente es el trabajo colaborativo, con un espíritu reflexivo, en el que se debe analizar, supervisar y modificar la práctica en la acción, además de tener claros los contenidos y objetivos a lograr en las diferentes propuestas.

Figura 4. Página donde se muestran los temas a debatir por los miembros de la red



\section{¿Hacia dónde vamos?}

No olvidemos que la educación está actualmente preocupada por modernizar sus métodos y por aprovechar los recursos más novedosos de comunicación vía satélite y de computación -las llamadas nuevas tecnologías-. Porque ciertamente, aplicaciones como la consulta de datos a través de una navegación por la Internet, el intercambio individual y colectivo por correo electrónico y las teleconferencias y videoconferencias interactivas por vía satélite son una gran oportunidad manifiesta para la educación (Alonso, 2004).

A partir de la experiencia de trabajar en una plataforma, llegamos a la conclusión de que hemos desarrollado competencias y con ellas aprendido a trabajar en Internet, que sin lugar a dudas nos serán de gran utilidad 
en un futuro no muy lejano. Y esto, podremos aplicarlo en la vida cotidiana, para que además de las clases, le dediquemos un tiempo extra a la educación, trabajando con este medio tecnológico.

Hay muchos retos por delante pues no hemos logrado que todos los alumnos participen y nos preocupa ya que se necesita que haya una transformación en las mentalidades para que aprendan a usar la Internet. Tenemos una gran tarea para el siguiente ciclo con más tiempo para provocar que se dé un cambio, pero éste no debe ser sólo de forma sino también de fondo: una plataforma educativa es lo ideal para que los estudiantes desarrollemos competencias tecnológicas. Creemos que esto contribuiría muy significativamente en todos, además de que ayudaría a obtener un mejor desempeño y alcanzar de manera más fácil las metas que nos hemos propuesto.

Como futuros docentes está en nuestras manos una parte del porvenir educativo, y es por eso que existe un reto que consideramos importante y oportuno: aprender a desarrollar competencias en otros ámbitos como la red mundial, donde la tecnología ha invadido todos los ámbitos de la vida social, para convertirse en una parte de nuestra cotidianeidad.

\section{Conclusiones}

Finalmente, algunos comentarios relacionados con el trabajo en la red. En primer lugar nos ha servido como experiencia formativa novedosa y confiamos en que sirva a otros estudiantes y docentes para prepararse en el uso y aprovechamiento de las herramientas necesarias para el desarrollo del trabajo docente. En la red se han sorteado algunas dificultades $\mathrm{y}$ hemos encontrado un apoyo importante para trabajar nuestras competencias tecnológicas. Ante la efervescente y siempre cambiante dinámica social, las necesidades de formación de los docentes se prolongan más allá de los primeros estudios de la licenciatura y se extienden a lo largo de toda la trayectoria laboral, ya que la formación continua resulta imprescindible, tanto por las exigencias derivadas de los cambios en los entornos laborales como también para hacer frente a los cambios que se producen en nuestros contextos, a la par de que crece la importancia de la educación informal a través de los medios de comunicación y muy especialmente Internet, pues la cantidad de tiempo que las personas les dedican y las infinitas posibilidades de acceso que proporcionan hacen de ellos una de las principales fuentes de información y por supuesto todo esto exige nuevas competencias profesionales para los formadores.

\section{Bibliografía}

Alonso, A. (2004). Los medios en la comunicación educativa. México, Limusa, Noriega Editores.

Andrade, R. y Sánchez, A. (2010). Las competencias en los entornos virtuales. Memoria académica del Primer Congreso Latinoamericano de Ciencias de la Educación. Recuperado el 24 de marzo de 2011 de http://fch.mxl.uabc.mx/lateduca/ lateduca.htm.

Bazdresch, M. (2005). Las competencias en la Formación de Docentes. Revista Educar de Jalisco, 35, Octubre-Diciembre. México. Recuperado el 24 de marzo de 2009 de http://educar.jalisco.gob.mx/35/Educar\%20No\%2035baja.pdf .

Cabrero, J. y Llorente, M. (2008). La alfabetización digital de los alumnos. Competencias digitales para el siglo XXI. Revista Portuguesa de Pedagogía, 42 (2), 728 (ISSN: 0870418). Recuperado el 13 de abril de 2011 de http://tecnologiaedu. us.es/cuestionario/bibliovir/jca26.pdf .

Chan, M. (2005). Competencias mediacionales para la educación en línea. Revista Electrónica de Investigación Educativa 2, (7). Universidad Autónoma de Baja California, Ensenada, México. Recuperado el 18 de marzo de 2011 de http://redie.uabc.mx/vol7no2/contenido-chan.html.

Coll, C. (1992). Los contenidos en la reforma: enseñanza y aprendi- 
zaje de conceptos, procedimientos y actitudes, España, Santillana.

Fernández, R. (2003). Competencias profesionales del docente en la sociedad del SIGLO XXI. Revista Organización y Gestión Educativa, 11 (1), 100. España, Ciss Praxis. Recuperado el 10 de marzo de 2009 de http://www.uclm.es/profesorado/ Ricardo/Cursos/CompetenciaProfesionales.pdf.

Guitert, M., Romeau, T. y Pérez-Mateo, M. (2007). Competencias TIC y trabajo en equipo en entornos virtuales. Revista de Universidad y Sociedad del Conocimiento, 1 (4), 1-12 Recuperado el 20 de mayo de 2011 de http://www.uoc.edu/ rusc/4/1/dt/esp/guitert_romeu_perez-mateo.pdf .

Hernández, M. y González, M. (2005). Los objetos de aprendizaje reutilizables (OAR): Modificaciones en torno a la configuración del conocimiento pedagógico y otras competencias exigidas en la sociedad de la información. RED Revista de Educación a Distancia, 3 (4), 1-13. España. Recuperado el 15 de junio de 2011 de http://redalyc.uaemex.mx/src/inicio/ArtPdfRed.jsp?iCve=54709605.

López, M. y Flores, K. (2006). Análisis de competencias a partir del uso de las TIC. Apertura: Revista de Innovación, 5 (6). Universidad de Guadalajara, México. Recuperado el 10 de enero de $2011 \mathrm{de}$ http://www.udgvirtual.udg.mx/apertura/num5/pdfs/analisis_competencias.pdf.
Schmelkes, S. (1994). Hacia una mejor calidad de nuestras escuelas. México. OEA.

SEP (1997). Plan de estudios. Licenciatura en educación primaria. México, SEP.

SEP (1999). Plan de estudios. Licenciatura en educación secundaria. México, SEP.

Sobrado, L. (2006). Las competencias de los orientadores en el ámbito de las TIC (Tecnologías de la Información y de la Comunicación): diagnóstico y desarrollo. Estudios Sobre Educación, 11 (8), 27-43. Publicaciones de la Universidad de Navarra, España. Recuperado el 22 de abril de 2011 de http://dspace.unav.es/dspace/bitstream/10171/8957/1/Eb.pdf .

UNESCO (2005). Hacia las sociedades del conocimiento. Publicaciones de la UNESCO. Recuperado el 28 de enero de 2012 de http://unesdoc.unesco.org/images/0014/001419/141908s. pdf

UNESCO (2008). Estándares de competencias en TIC para docentes. Publicaciones de la UNESCO. Recuperado el 11 de mayo de 2011 de http://www.eduteka.org/EstandaresDocentes Unesco.php.

Torres, R. M. (2001). La formación en la era de la informática y la lucha contra la pobreza. ED-01/PROMEDLAC VII/ Documento de Apoyo. Recuperado el 29 de agosto de 2009 de http://usuarios.lycos.es/hcalibra/OEI/torres.pdf. 